\title{
Eigenvalues of Hermitian Toeplitz matrices with polynomially increasing entries
}

\author{
J. M. Bogoya, A. Böttcher, and S. M. Grudsky ${ }^{1}$ \\ Dedicated to William F. Trench and Harold Widom \\ on their $80^{\text {th }}$ birthdays
}

\begin{abstract}
The paper is concerned with finite Hermitian Toeplitz matrices whose entries in the first row grow like a polynomial. Such matrices cannot be viewed as truncations of an infinite Toeplitz matrix which is generated by an integrable function or a nice measure. The main results describe the first-order asymptotics of the extreme eigenvalues as the matrix dimension goes to infinity and also deliver unexpected barriers for the eigenvalues. One purpose of the paper is to popularize once more that questions on the eigenvalues of matrices can be answered in a very elegant way by passing to integral operators. This idea was introduced by Harold Widom about fifty years ago. In this way one can also give an alternative proof to results by William F. Trench on Hermitian Toeplitz matrices with increasing entries.
\end{abstract}

Mathematics Subject Classification (2010). Primary 15B05; Secondary 15A18, 15B57, $45 \mathrm{C} 05,47 \mathrm{~N} 40$.

Keywords. Toeplitz matrix, integral operator, circulant matrix, eigenvalue.

\section{Introduction and main results}

Given a real number $a_{0}$ and $n-1$ complex numbers $a_{1}, \ldots, a_{n-1}$, we denote by $T_{n}=T_{n}\left[a_{0}, a_{1}, \ldots, a_{n-1}\right]$ the Hermitian $n \times n$ Toeplitz matrix whose first row is constituted by these numbers,

$$
T_{n}=T_{n}\left[a_{0}, a_{1}, \ldots, a_{n-1}\right]=\left(\begin{array}{llll}
a_{0} & a_{1} & \ldots & a_{n-1} \\
\bar{a}_{1} & a_{0} & \ldots & a_{n-2} \\
\vdots & \vdots & \ddots & \vdots \\
\bar{a}_{n-1} & \bar{a}_{n-2} & \ldots & a_{0}
\end{array}\right) .
$$

\footnotetext{
${ }^{1}$ S. M. Grudsky acknowledges support by CONACYT grant 102800.
} 
We are interested in the behavior of the eigenvalues of $T_{n}$. Much is known if the numbers $a_{k}$ are the Fourier coefficients of an $L^{1}$ function or of a Radon measure. In the first case the numbers $a_{k}$ converge to zero as $k \rightarrow \infty$, while in the second they must necessarily remain bounded. We here study the case where the numbers $a_{k}$ are equidistant sample values of a polynomial,

$$
a_{k}=p_{0}+p_{1} k+\cdots+p_{\alpha-1} k^{\alpha-1}+k^{\alpha}, \quad \alpha \in \mathbb{N},
$$

or, more generally,

$$
a_{k}=k^{\alpha}+o\left(k^{\alpha}\right), \quad \alpha \in(0, \infty) .
$$

This paper was originally motivated by an observation of Stephan Garcia. He detected numerically that the symmetric Toeplitz matrices

$$
T_{n}[R, R-h, R-2 h, \ldots, R-(n-1) h]
$$

are positive definite as long as all entries are positive and asked for a mathematical explanation of this phenomenon. Figure 1 shows an example and even reveals that the matrices remain positive definite also when some of its entries become negative provided the sum of the entries is still positive.

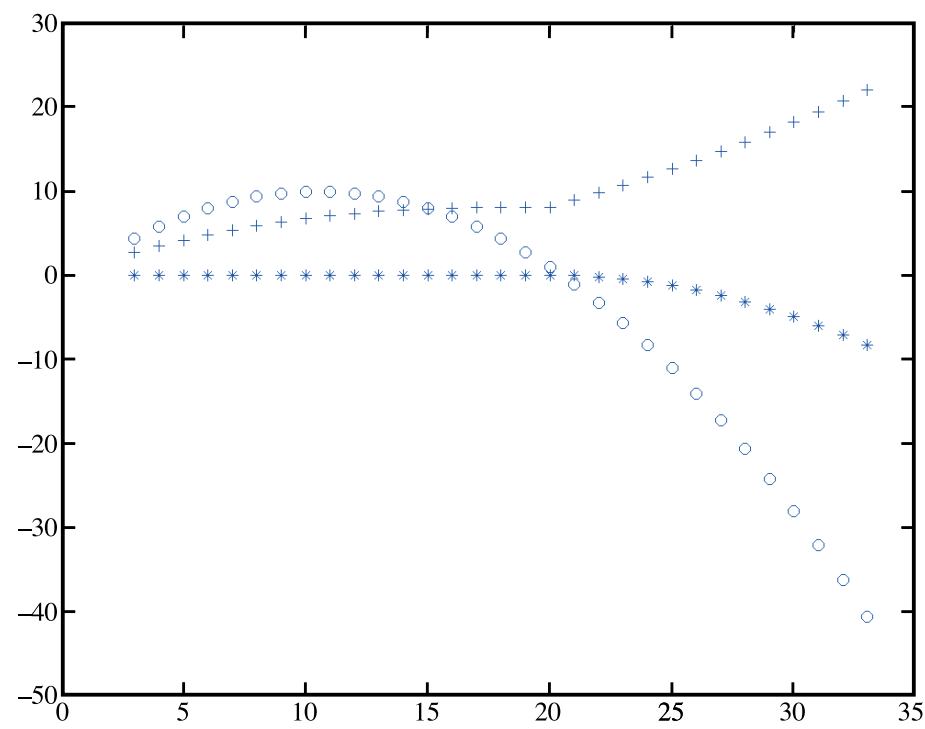

Figure 1. The minimal eigenvalues (asterisks) and the maximal eigenvalues (pluses) of $T_{n}[1,1-h, 1-2 h, \ldots, 1-(n-1) h]$ with $h=1 / 10$ for $3 \leq n \leq 33$ and the sum $s_{n}=2 n-1-h n(n-1)$ of the entries in the first row and first column (circles). The minimal eigenvalue is greater than 0.05 for $n \leq 20$ and negative for $n \geq 21$. We have $s_{n} \geq 1$ for $n \leq 20$ and $s_{n} \leq-1$ for $n \geq 21$. 
One is tempted to think of $T_{n}[1,1-h, 1-2 h, \ldots, 1-(n-1) h]$ as the $n \times n$ Toeplitz matrix which is induced by the symbol

$$
u_{n}(\varphi) \stackrel{\text { def }}{=} 1+\sum_{k=1}^{n-1}(1-k h)\left(e^{k i \varphi}+e^{-k i \varphi}\right)=1+2 \sum_{k=1}^{n-1}(1-k h) \cos (k \varphi)
$$

and then to show that $u_{n}(\varphi)>0$ for $\varphi \in[0,2 \pi)$. However, as already noticed by Stephan Garcia and as seen in Figure 2, this is not true. We will prove that the positive definiteness of the matrices is in fact a consequence of the circumstance that $u_{n}\left(\varphi_{\ell}\right)>0$ for $\varphi_{\ell}=2 \pi \ell /(2 n-1), \ell=1,2, \ldots, 2 n-2$.

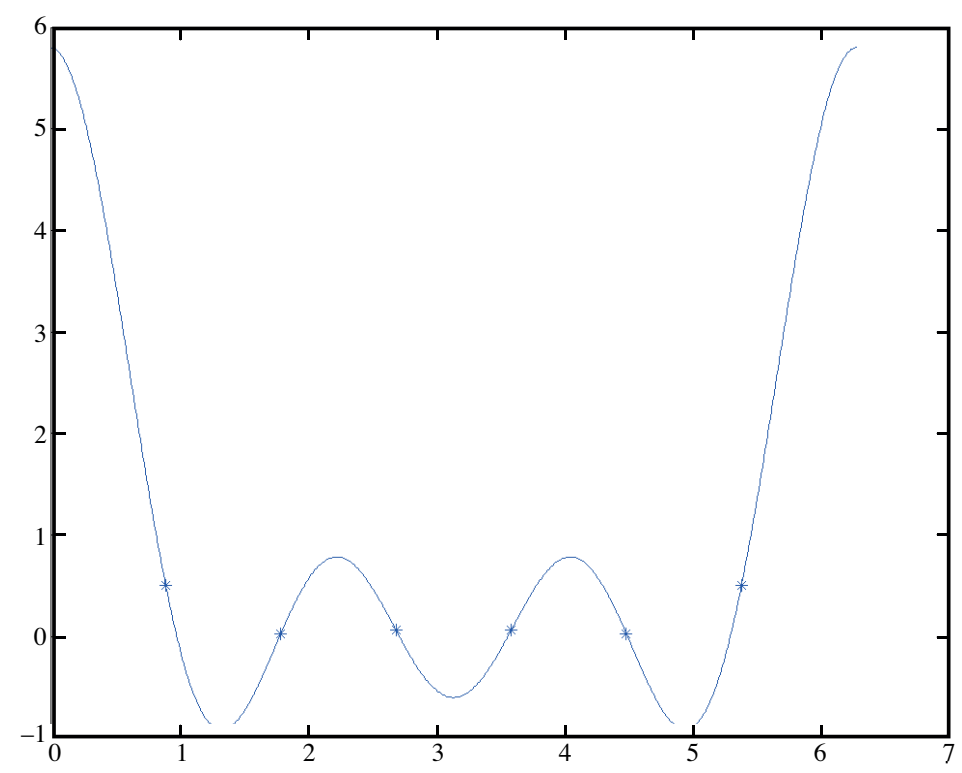

Figure 2. The graph of the symbol function $1+2(1-h) \cos \varphi+2(1-2 h) \cos (2 \varphi)+$ $2(1-2 h) \cos (3 \varphi)(h=1 / 10, n=4)$. The asterisks are the values of the function at $\varphi_{\ell}=$ $2 \pi \ell / 7$.

Another observation from Figure 1 is that for large $n$ the extreme eigenvalues of $T_{n}$ seem to move to infinity. Figures 3 to 5 contain more examples and indicate that the extreme eigenvalues are asymptotically equal to a constant times $n^{\alpha+1}$. We will prove this as well. Figure 3 also indicates that with the exception of the largest eigenvalue all eigenvalues are negative and that they cluster close to zero before starting the movement to minus infinity. In other words, Figure 3 makes us surmise that the negative eigenvalues are asymptotically of the form $-c_{\ell} n^{2}$ with very small positive coefficients $c_{\ell}$. All this will be proved. The matrices $T_{n}$ of Figure 4 will be shown to have rank 3 , and hence $n-3$ eigenvalues are exactly zero, which is also lucidly seen in Figure 4. 


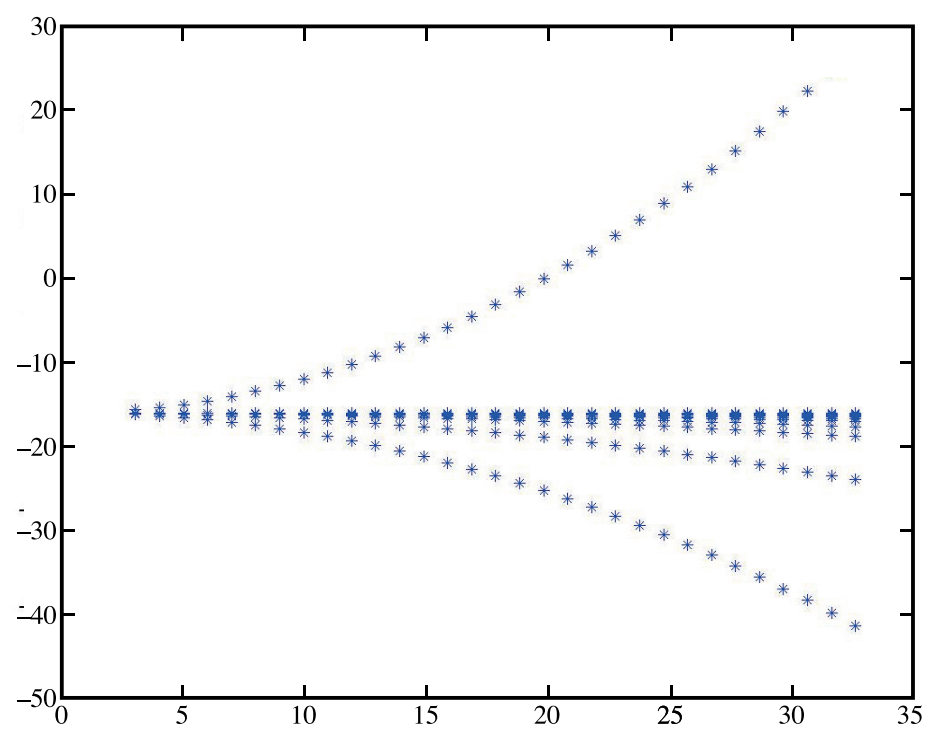

Figure 3. The eigenvalues of $T_{n}[0,1,2, \ldots, n-1]$ for $3 \leq n \leq 33$.

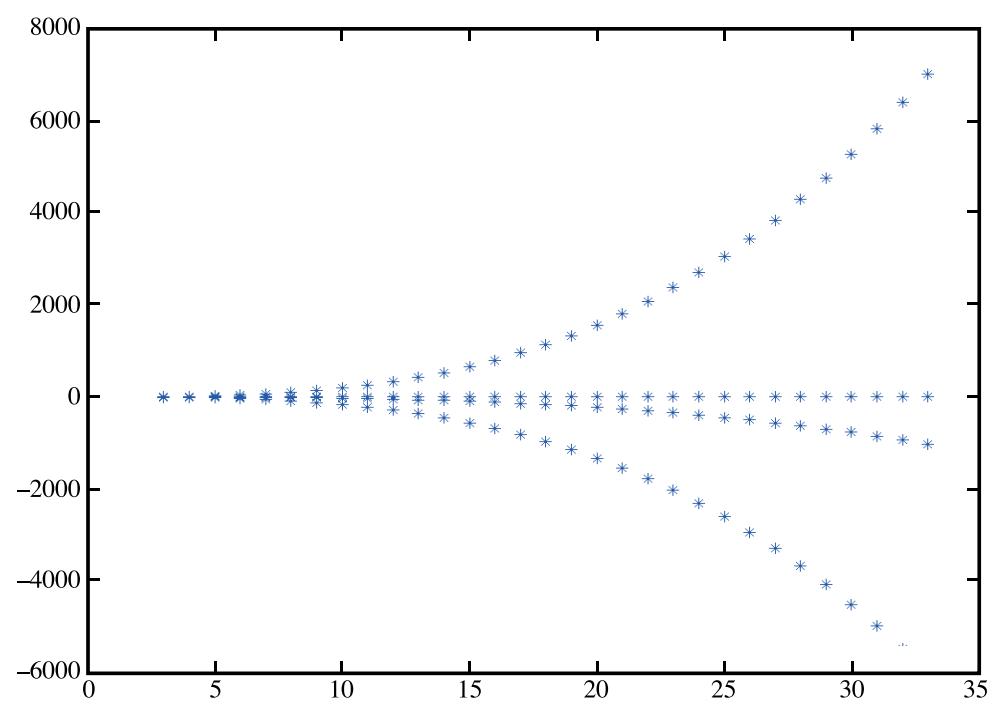

Figure 4. The eigenvalues of $T_{n}\left[0,1^{2}, 2^{2}, \ldots,(n-1)^{2}\right]$ for $3 \leq n \leq 33$. 
Herewith our main results. We denote the eigenvalues of $T_{n}$ by

$$
\lambda_{1}\left(T_{n}\right) \leq \lambda_{2}\left(T_{n}\right) \leq \ldots \leq \lambda_{n}\left(T_{n}\right) .
$$

We also abbreviate $T_{n}\left[0,1^{\alpha}, 2^{\alpha}, \ldots,(n-1)^{\alpha}\right]$ to $T_{n, \alpha}$ and refer to these matrices as the canonical matrices. Let $K_{\alpha}$ be the integral operator on $L^{2}(0,1)$ given by

$$
\left(K_{\alpha} f\right)(x)=\int_{0}^{1}|x-y|^{\alpha} f(y) d y, \quad x \in(0,1) .
$$

The operator $K_{\alpha}$ is compact and selfadjoint. For $\alpha>0$, it is even a trace class operator with the trace

$$
\operatorname{tr} K_{\alpha}=\int_{0}^{1}|x-x|^{\alpha} d x=0 .
$$

We denote its positive eigenvalues by

$$
\mu_{1}\left(K_{\alpha}\right) \geq \mu_{2}\left(K_{\alpha}\right) \geq \ldots>0
$$

and write

$$
v_{1}\left(K_{\alpha}\right) \leq v_{2}\left(K_{\alpha}\right) \leq \ldots<0
$$

for its negative eigenvalues, all counted with their multiplicity. Finally, we let $\mathscr{L}_{+}$ stand for the index set of the positive eigenvalues, that is, $\mathscr{L}_{+}=\{1,2, \ldots, m\}$ if $K_{\alpha}$ has exactly $m<\infty$ positive eigenvalues, and $\mathscr{L}_{+}=\mathbb{N}$ if the operator has countably many positive eigenvalues. The index set $\mathscr{L}_{-}$is defined analogously. By virtue of (3), the sets $\mathscr{L}_{+}$and $\mathscr{L}_{-}$are not empty.

Theorem 1.1. Let $\alpha>0$ be a real number, suppose $a_{k}=k^{\alpha}+o\left(k^{\alpha}\right)$ as $k \rightarrow \infty$, and consider $T_{n}=T_{n}\left[a_{0}, a_{1}, \ldots, a_{n-1}\right]$. Then, as $n \rightarrow \infty$,

$$
\begin{aligned}
\lambda_{n+1-\ell}\left(T_{n}\right) & =\mu_{\ell}\left(K_{\alpha}\right) n^{\alpha+1}+o\left(n^{\alpha+1}\right) \quad \text { for } \ell \in \mathscr{L}_{+}, \\
\lambda_{n+1-\ell}\left(T_{n}\right) & =o\left(n^{\alpha+1}\right) \quad \text { for } \ell \in \mathbb{N} \backslash \mathscr{L}_{+}, \\
\lambda_{\ell}\left(T_{n}\right) & =v_{\ell}\left(K_{\alpha}\right) n^{\alpha+1}+o\left(n^{\alpha+1}\right) \text { for } \ell \in \mathscr{L}_{-}, \\
\lambda_{\ell}\left(T_{n}\right) & =o\left(n^{\alpha+1}\right) \quad \text { for } \ell \in \mathbb{N} \backslash \mathscr{L}_{-} .
\end{aligned}
$$

If $a_{k}$ is given by (1), then (4) and (6) hold with $O\left(n^{\alpha}\right)$ in place of $o\left(n^{\alpha+1}\right)$.

The following two theorems provide us with more precise information for $\alpha=1$.

Theorem 1.2. (a) The operator $K_{1}$ has exactly one positive eigenvalue $\mu_{1}\left(K_{1}\right)$ and countably many negative eigenvalues. All these eigenvalues have the multiplicity 1. The positive eigenvalue of $K_{1}$ is

$$
\mu_{1}\left(K_{1}\right)=\frac{2}{\omega_{0}^{2}}=0.3471 \ldots
$$


where $\omega_{0}$ is the positive solution of the equation $2+2 \cosh \omega-\omega \sinh \omega=0$, and the negative eigenvalues are

$$
v_{\ell}\left(K_{1}\right)=-\frac{2}{\omega_{\ell}^{2}}
$$

where $0<\omega_{1}<\omega_{2}<\ldots$ are the positive solutions of the equation $2+2 \cos \omega+$ $\omega \sin \omega=0$. In particular,

$$
v_{1}\left(K_{1}\right)=-\frac{2}{\pi^{2}}, \quad v_{3}\left(K_{1}\right)=-\frac{2}{(3 \pi)^{2}}, \quad v_{5}\left(K_{1}\right)=-\frac{2}{(5 \pi)^{2}}, \quad \ldots
$$

(b) For the canonical matrices $T_{n, 1}$, we have $\lambda_{n-1}\left(T_{n, 1}\right) \leq-1 / 4$ for all $n \geq 2$.

The first four significant digits of the numerical values of the smallest negative eigenvalues of $K_{1}$ are

$$
\begin{array}{|c|c|c|c|c|c|}
v_{1}\left(K_{1}\right) & v_{2}\left(K_{1}\right) & v_{3}\left(K_{1}\right) & v_{4}\left(K_{1}\right) & v_{5}\left(K_{1}\right) & v_{6}\left(K_{1}\right) \\
\hline-0.2026 & -0.0638 & -0.0225 & -0.0133 & -0.0081 & -0.0058
\end{array} .
$$

Numerical experiments show that actually $\lambda_{n-1}\left(T_{n, 1}\right)<-1 / 2$. We have not been able to prove this.

Figure 5 illustrates that the asymptotics of Theorem 1.1 with the constants from Theorem 1.2(a) deliver very good approximations even for small $n$.

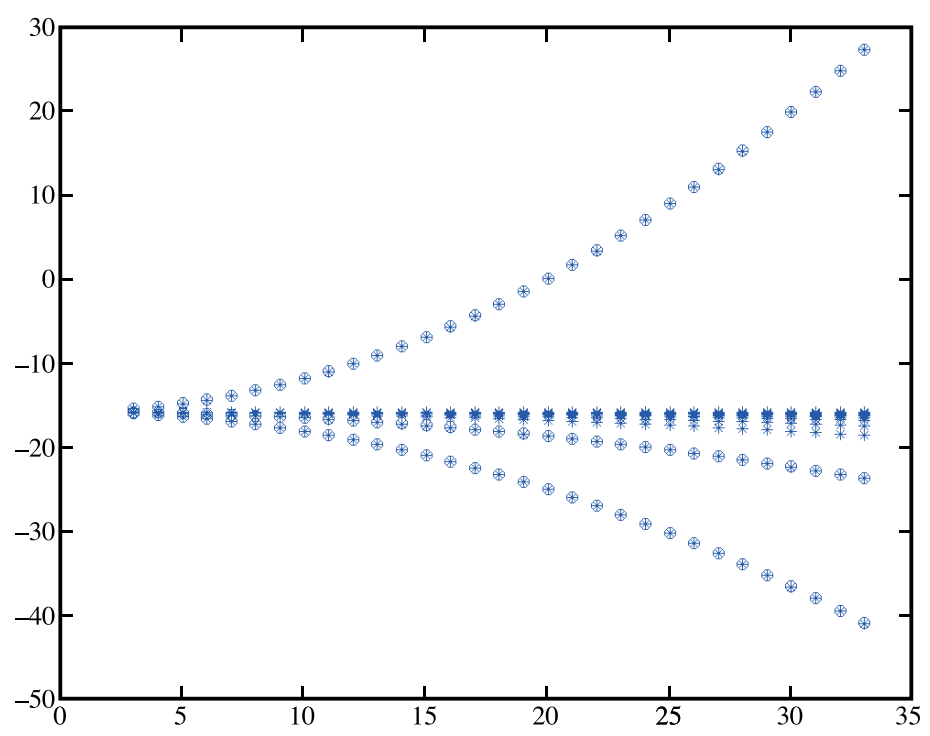

Figure 5. The eigenvalues of $T_{n, 1}=T_{n}[0,1,2, \ldots, n-1]$ for $3 \leq n \leq 33$ as in Figure 3 (asterisks) and the values $\mu_{1}\left(K_{1}\right) n^{2}, v_{1}\left(K_{1}\right) n^{2}, v_{2}\left(K_{1}\right) n^{2}$ (circles). 
The following is a restatement of parts of Theorems 1.1 and 1.2. It proves what we see in Figure 1.

Theorem 1.3. Let $h>0$ and $T_{n}=T_{n}[R, R-h, \ldots, R-(n-1) h]$.

(a) We have $\lambda_{1}\left(T_{n}\right) \geq h / 4$ whenever the sum $s_{n}=R(2 n-1)-h n(n-1)$ of the entries in the first row and the first column of $T_{n}$ satisfies $s_{n} \geq h / 4$.

(b) The smallest and largest eigenvalues of $T_{n}$ satisfy

$$
\lambda_{1}\left(T_{n}\right)=-h \frac{2}{\omega_{0}^{2}} n^{2}+O(n), \quad \lambda_{n}\left(T_{n}\right)=h \frac{2}{\pi^{2}} n^{2}+O(n),
$$

where $\omega_{0}$ is as in Theorem 1.2.

Things are especially nice in the case where $\alpha$ is an even integer. The following theorem was essentially established by Trench in [19].

Theorem 1.4 (Thench). Let $\alpha$ be an even natural number.

(a) The operator $K_{\alpha}$ has exactly $\alpha+1$ nonzero eigenvalues,

$$
v_{1}\left(K_{\alpha}\right)<\ldots<v_{q}\left(K_{\alpha}\right)<0<\mu_{p}\left(K_{\alpha}\right)<\ldots<\mu_{1}\left(K_{\alpha}\right), \quad p+q=\alpha+1,
$$

and these eigenvalues are the eigenvalues of the matrix

$$
M_{\alpha}=\left((-1)^{\alpha-j}\left(\begin{array}{l}
\alpha \\
j
\end{array}\right) \frac{1}{\alpha+1-j+k}\right)_{j, k=0}^{\alpha} .
$$

(b) The rank of the canonical matrices $T_{n, \alpha}$ is $\alpha+1$ for all $n \geq \alpha+1$ and

$$
\begin{aligned}
\lambda_{n+1-\ell}\left(T_{n, \alpha}\right) & =\mu_{\ell}\left(K_{\alpha}\right) n^{\alpha+1}+O\left(n^{\alpha}\right) \text { for } \ell=1, \ldots, p, \\
\lambda_{\ell}\left(T_{n, \alpha}\right) & =v_{\ell}\left(K_{\alpha}\right) n^{\alpha+1}+O\left(n^{\alpha}\right) \quad \text { for } \ell=1, \ldots, q, \\
\lambda_{q+1}\left(T_{n, \alpha}\right) & =\ldots=\lambda_{n-p}\left(T_{n, \alpha}\right)=0 \quad \text { for } n \geq \alpha+1 .
\end{aligned}
$$

Straightforward computation gives that the eigenvalues of $M_{2}$ are

$$
\begin{aligned}
& \nu_{1}\left(K_{2}\right)=-\frac{1}{6}=-0.1666 \ldots, \\
& \nu_{2}\left(K_{2}\right)=\frac{1}{12}-\frac{\sqrt{5}}{20}=-0.0285 \ldots, \\
& \mu_{1}\left(K_{2}\right)=\frac{1}{12}+\frac{\sqrt{5}}{20}=0.1951 \ldots
\end{aligned}
$$


This is in perfect accordance with Figure 4, which shows the eigenvalues of $T_{n, 2}$. Numerically we obtained that, showing only the first four significant digits, the eigenvalues of $M_{4}$ are

$$
-0.1046, \quad-0.0070, \quad 0.0010, \quad 0.0046, \quad 0.1059,
$$

while those of $M_{6}$ equal

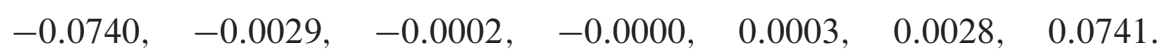

For odd integers $\alpha$, the determination of the eigenvalues of $K_{\alpha}$ is in principle possible, but the computations are very expensive. We will say more about this issue in Section 5. We confine ourselves to the following.

Theorem 1.5. Let $\alpha>0$ be a real number. Then $\left|v_{1}\left(K_{\alpha}\right)\right| \leq \mu_{1}\left(K_{\alpha}\right)$, the largest eigenvalue $\mu_{1}\left(K_{\alpha}\right)$ satisfies $C_{\alpha} \leq \mu_{1}\left(K_{\alpha}\right) \leq D_{\alpha}$ with

$$
C_{\alpha}=\frac{\sqrt{2}}{\alpha+1} \sqrt{\frac{1}{2 \alpha+3}+\frac{\Gamma(\alpha+2)^{2}}{\Gamma(2 \alpha+4)}},
$$

and

$$
D_{\alpha}=\frac{1}{\sqrt{(\alpha+1)(2 \alpha+1)}}
$$

and the function $\alpha \mapsto \mu_{1}\left(K_{\alpha}\right)$ is monotonically decreasing.

Table 1 exhibits the estimates for some values of $\alpha$, showing only the first four digits of the numbers. The value $\mu_{1}\left(K_{1}\right)=0.3471$ is from Theorem 1.2(a), the remaining values for $\mu_{1}\left(K_{\alpha}\right)$ are guesses based on computing $\lambda_{n}\left(T_{n, \alpha}\right) / n^{\alpha+1}$ for some large $n$.

Table 1. Estimates of $C_{\alpha}, \mu_{1}\left(K_{\alpha}\right)$, and $D_{\alpha}$.

\begin{tabular}{|c|c|c|c|}
$\alpha$ & $C_{\alpha}$ & $\mu_{1}\left(K_{\alpha}\right)$ & $D_{\alpha}$ \\
\hline 1 & 0.3416 & 0.3471 & 0.4082 \\
3 & 0.1187 & 0.1363 & 0.1890 \\
5 & 0.0654 & 0.0871 & 0.1231 \\
7 & 0.0429 & 0.0645 & 0.0913 \\
9 & 0.0309 & 0.0512 & 0.0725
\end{tabular}




\section{Notes}

The pioneering results on the asymptotic behavior of the eigenvalues of Hermitian Toeplitz matrices go back to Kac, Murdock, and Szegó; see paper [10] and the book [9]. They studied the collective behavior of the eigenvalues, that is, the sum

$$
\frac{1}{n} \sum_{k=1}^{n} F\left(\lambda_{k}\left(T_{n}\right)\right)
$$

for certain test functions $F$, and also the behavior of the extreme eigenvalues $\lambda_{\ell}\left(T_{n}\right)$ and $\lambda_{n+1-\ell}\left(T_{n}\right)$. Their basic assumption was that $T_{n}=T_{n}\left[a_{0}, a_{1}, \ldots, a_{n-1}\right]$ where $\left\{a_{k}\right\}$ is the sequence of the Fourier coefficients of a smooth function $a$ on the unit circle.

For the extreme eigenvalues, two major problems were left. The first concerns higher-order asymptotics for smooth functions $a$. This problem was thoroughly investigated by Parter [12], [13], and Widom [23], [24], [25]. Part of these two hero's work is outlined in more detail on pages 256-259 of [4]. Recent work on this topic includes the papers [11], [29]. We also note that paper [5] contains higher-order asymptotics for the individual eigenvalues $\lambda_{k}\left(T_{n}\right)$ inside the spectrum, e.g. for $k / n \rightarrow x \in[0,1]$, however, under the assumption that $a$ is a Laurent polynomial which is subject to additional conditions. The second problem was the passage from smooth functions $a$ to more general functions, and here it was Serra Capizzano [15] and [16] who was able to prove first-order results under the sole assumption that $a$ be an $L^{1}$ function.

It took significant efforts to come up with (11) in more general situations. The limit of (11) was determined by Parter [14] for $a \in L^{\infty}$, by Tyrtyshnikov [20] and [21] for $a \in L^{2}$, and then by Zamarashkin and Tyrtyshnikov [28] for $a \in L^{1}$. Eventually, Tyrtyshnikov and Zamarashkin succeeded in determining the limit of (11) in the case where $\left\{a_{k}\right\}$ is the sequence of the Fourier coefficients of a Radon measure.

In all the situations described above, $a_{k}=O(1)$. Trench [18] and [19] considered matrices in which

$$
a_{k}=\sum_{j=1}^{m} P_{j}(|k|) \zeta_{j}^{k}
$$

where $P_{j}$ are polynomials and $\zeta_{j}$ are complex numbers. Clearly, if $\left|\zeta_{j}\right| \geq 1$, then the entries do not remain bounded. Trench proved that the spectrum of $T_{n}$ is the union of a "distributed" part, in which sums like (11) have a limit, and an "outlying" part, which consists of eigenvalues that move to $\pm \infty$. In the case where $m=1$, $\zeta_{1}=1$, and $P_{1}(k)=k^{\alpha}$, Trench's matrices become our canonical matrices $T_{n, \alpha}$, and if, in addition, $\alpha$ is an even natural number, he established Theorem 1.4. He did not consider the integral operator $K_{\alpha}$ but rather introduced the coefficients $\mu_{k}\left(K_{\alpha}\right)$ and $v_{k}\left(K_{\alpha}\right)$ directly as the eigenvalues of the matrix $M_{\alpha}$. Note that the Toeplitz matrix $\left((j-k)^{\alpha}\right)_{j, k=1}^{n}(\alpha \in \mathbb{N})$ is Hermitian if and only if $\alpha$ is even, in which case $(j-k)^{\alpha}=|j-k|^{\alpha}$. 
Widom [26] studied Wiener-Hopf integral operators over $(0, \tau)$ under the assumption that the kernel $\kappa(t)$ grows more rapidly than every polynomial. He showed that the two extremal eigenvalues of the operator are asymptotically equal to $\pm \kappa(2 \tau)^{2} / 2 \kappa^{\prime}(2 \tau)$ and that the other eigenvalues are $o\left(\kappa(2 \tau)^{2} / 2 \kappa^{\prime}(2 \tau)\right)$ as $\tau$ goes to $\infty$. Note that for Wiener-Hopf integral operators the case of polynomial kernels is trivial: making the change of variables $t=\tau x, s=\tau y$, we see that the eigenvalues of the operator given by

$$
\left(W_{\tau} f\right)(t)=\int_{0}^{\tau}|t-s|^{\alpha} f(s) d s, \quad t \in(0, \tau),
$$

are $\tau^{\alpha+1}$ times the eigenvalues of the operator $K_{\alpha}$ defined by (2). Wiener-Hopf integral operators are the continuous analog of Toeplitz matrices, and the operators of [26] are the analog of Toeplitz matrices $T_{n}\left[a_{0}, a_{1}, \ldots, a_{n-1}\right]$ with $a_{k}=e^{\gamma_{k}}$ where $\gamma_{k}$ increases more rapidly than $\log k$.

There are many techniques to treat eigenvalues of Toeplitz matrices; see, for example, [6]. We here employ two of them, which, curiously, are not discussed in [6]. The first is based on replacing the matrix $\left(a_{j, k}\right)_{j, k=0}^{n-1}$ by the integral operator on $L^{2}(0,1)$ whose kernel is $k(x, y)=a_{[n x],[n y]}$, where [·] denotes the integral part. This is an old idea, which was heavily exploited for probably the first time by Widom [23], [24], [25], [27] and independently by Shampine [17]. The recent papers [1], [2], [3], [7], and [8] illustrate the power of this idea in several contexts.

The second idea consists in relating the eigenvalues of Toeplitz matrices with those of cleverly constructed circulant matrices. One choice is the circulant matrix which is the best approximation to the Toeplitz matrix in the Frobenius norm. This method is prevailing in the works by Serra Capizzano, Trench, Tyrtyshnikov, and Zamarashkin, for example. Another well known choice is based on bordering the $n \times n$ Toeplitz matrix to a $(2 n-1) \times(2 n-1)$ circulant matrix and on exploiting Cauchy's interlace theorem. The latter method was used in [29], for instance, and will be exerted here, too.

We remark that the case $\alpha=0$, that is, the case where $a_{k}=1+o(1)$, does not cause problems. The integral operator $K_{0}$ is the rank-one operator given by $\left(K_{0} f\right)(x)=\int_{0}^{1} f(y) d y$, and in contrast to the case $\alpha>0$, the trace of $K_{0}$ is not zero, so that we cannot guarantee the existence of both a positive and a negative eigenvalue. It is easily seen that the only eigenvalue of $K_{0}$ is 1 , the multiplicity being one. Clearly, $\lambda_{n}\left(T_{n}[1,1, \ldots, 1]\right)=n$ and $\lambda_{k}\left(T_{n}[1,1, \ldots, 1]\right)=0$ for $1 \leq k \leq n-1$. For $\alpha=0$, Theorem 1.1 amounts to saying that $\lambda_{n}\left(T_{n}\right)=n+o(n)$ and $\lambda_{k}\left(T_{n}\right)=o(n)$ for $1 \leq k \leq n-1$.

The rest of the paper contains the proofs of Theorems 1.1 to 1.5 . Figure 2 is addressed in Remark 4.3, and further results are provided by Remarks 4.2, 5.1, and 5.2. 


\section{Matrices versus integral operators}

This section is devoted to the proof of Theorem 1.1. The following result is due to Widom [23], [24], [25], [27], and Shampine [17].

Lemma 3.1 (Widom and Shampine). Let $A=\left(a_{i j}\right)_{i, j=0}^{n-1}$ be a matrix in $\mathbb{C}^{n \times n}$ and let $G$ be the integral operator on $L^{2}(0,1)$ given by

$$
(G f)(x)=\int_{0}^{1} a_{[n x],[n y]} f(y) d y, \quad x \in(0,1),
$$

where $[\cdot]$ denotes the integral part. Then a nonzero complex number $\lambda$ is an eigenvalue of $A$ of a certain algebraic multiplicity if and only if $\lambda / n$ is an eigenvalue of $G$ of the same algebraic multiplicity.

Proof. Let $J_{k}$ be the interval $(k / n,(k+1) / n)$, denote by $\chi_{k}$ the characteristic function of $J_{k}$, and consider the operators

$$
\begin{aligned}
S: \quad \mathbb{C}^{n} & \longrightarrow L^{2}(0,1), \\
\left(z_{k}\right)_{k=0}^{n-1} & \longmapsto \sqrt{n} \sum_{k=0}^{n-1} z_{k} \chi_{k}, \\
R: L^{2}(0,1) & \longrightarrow \mathbb{C}^{n}, \\
f & \longmapsto\left(\sqrt{n} \int_{J_{k}} f(x) d x\right)_{k=0}^{n-1} .
\end{aligned}
$$

It is easily seen that

$$
R S=I, \quad S^{*}=R, \quad n G=S A R
$$

It follows that $L^{2}(0,1)=\operatorname{Ran} S \oplus(\operatorname{Ran} S)^{\perp}=\operatorname{Ran} S \oplus \operatorname{Ker} R$ and that if $f=$ $g+v$ with $g \in \operatorname{Ran} S$ and $v \in \operatorname{Ker} R$, then $n G f=S A R(g+v)=S A R g$ with $S A R g \in \operatorname{Ran} S$. Consequently, the matrix representations of $n G$ and the identity operator $I$ with respect to the decomposition $L^{2}(0,1)=\operatorname{Ran} S \oplus \operatorname{Ker} R$ are

$$
n G=\left(\begin{array}{cc}
S A R & 0 \\
0 & 0
\end{array}\right), \quad I=\left(\begin{array}{cc}
S R & 0 \\
0 & I
\end{array}\right)
$$


Using elementary properties of operator determinants we therefore obtain

$$
\begin{aligned}
\operatorname{det}\left(I-\frac{n}{\lambda} G\right) & =\operatorname{det}\left(\begin{array}{cc}
S R-(1 / \lambda) S A R & 0 \\
0 & I
\end{array}\right) \\
& =\operatorname{det}\left(S R-\frac{1}{\lambda} S A R\right) \\
& =\operatorname{det} S\left(I-\frac{1}{\lambda} A\right) R \\
& =\operatorname{det} R S\left(I-\frac{1}{\lambda} A\right) \\
& =\operatorname{det}\left(I-\frac{1}{\lambda} A\right) .
\end{aligned}
$$

We now use this lemma in the case where $A=T_{n, \alpha}$ is the canonical matrix $T_{n}\left[0,1^{\alpha}, 2^{\alpha}, \ldots,(n-1)^{\alpha}\right]$. Let $G$ be the integral operator associated with $A$ as in Lemma 3.1. To emphasize the dependence on $n$ and $\alpha$, we write $G_{n, \alpha}$ for $G$. Recall the integral operator $K_{\alpha}$ given by (2). Finally, we put $K_{n, \alpha} \stackrel{\text { def }}{=} n^{-\alpha} G_{n, \alpha}$.

Lemma 3.2. The operators $K_{n, \alpha}$ converge to $K_{\alpha}$ uniformly, that is, in the norm.

Proof. The operator $K_{n, \alpha}-K_{\alpha}$ is the integral operator with the kernel

$$
\frac{|[n x]-[n y]|^{\alpha}}{n^{\alpha}}-|x-y|^{\alpha} .
$$

Writing $[n x]-[n y]=n(x-y)+\delta_{n}(x, y)$ with $\left|\delta_{n}(x, y)\right| \leq 2$, we see that (12) goes to zero uniformly in $(x, y) \in[0,1]^{2}$. This implies that $\left\|K_{n, \alpha}-K_{\alpha}\right\| \rightarrow 0$.

Lemma 3.3. As $n \rightarrow \infty$,

$$
\begin{aligned}
& \lambda_{n+1-\ell}\left(K_{n, \alpha}\right) \longrightarrow \mu_{\ell}\left(K_{\alpha}\right) \quad \text { for } \ell \in \mathscr{L}_{+}, \\
& \lambda_{n+1-\ell}\left(K_{n, \alpha}\right) \longrightarrow 0 \text { for } \ell \in \mathbb{N} \backslash \mathscr{L}_{+}, \\
& \lambda_{\ell}\left(K_{n, \alpha}\right) \longrightarrow v_{\ell}\left(K_{\alpha}\right) \quad \text { for } \ell \in \mathscr{L}_{-}, \\
& \lambda_{\ell}\left(K_{n, \alpha}\right) \longrightarrow 0 \text { for } \ell \in \mathbb{N} \backslash \mathscr{L}_{-} .
\end{aligned}
$$

Proof. We abbreviate $K_{n, \alpha}$ and $K_{\alpha}$ to $K_{n}$ and $K$, respectively.

Let $\ell \in \mathbb{N}$. There is an $M>0$ such that $M I+K_{n}$ is positive definite for all $n$. Consequently, the eigenvalues $\lambda_{n+1-\ell}\left(M I+K_{n}\right)$ are just the singular values $\sigma_{\ell}\left(M I+K_{n}\right)$. It follows that

$$
\lambda_{n+1-\ell}\left(K_{n}\right)=-M+\lambda_{n+1-\ell}\left(M I+K_{n}\right)=-M+\sigma_{\ell}\left(M I+K_{n}\right) .
$$


The $\ell$-th singular value is equal to the $\ell$-th approximation number, that is, to the distance to the set $\mathcal{F}_{\ell-1}$ of operators of rank at most $\ell-1$. Since $K_{n} \rightarrow K$ uniformly by Lemma 3.2, we may conclude that

$$
\operatorname{dist}\left(M I+K_{n}, \mathcal{F}_{\ell-1}\right) \longrightarrow \operatorname{dist}\left(M I+K, \mathscr{F}_{\ell-1}\right)=\sigma_{\ell}(M I+K)
$$

and hence

$$
\lambda_{n+1-\ell}\left(K_{n}\right) \longrightarrow-M+\sigma_{\ell}(M I+K) .
$$

If $\ell \in \mathscr{L}_{+}$, then $\sigma_{\ell}(M I+K)=\mu_{\ell}(M I+K)$ and we arrive at the conclusion that

$$
\lambda_{n+1-\ell}\left(K_{n}\right) \longrightarrow-M+\mu_{\ell}(M I+K)=\mu_{\ell}(K) .
$$

In the case where $\mathscr{L}_{+}=\mathbb{N}$, we are done.

So suppose $\mathscr{L}_{+}$is finite, $\mathscr{L}_{+}=\{1,2, \ldots, p\}$, and $\ell \geq p+1$. Then $\sigma_{\ell}(M I+K) \leq$ $M$ because we may ignore the $p$ largest eigenvalues and the remaining eigenvalues are all not greater than $M$. Therefore (18) implies that

$$
\limsup _{n \rightarrow \infty} \lambda_{n+1-\ell}\left(K_{n}\right) \leq 0 .
$$

Applying the previous argument to the operators $-K_{n}$, we analogously obtain that

$$
\lambda_{m}\left(K_{n}\right) \longrightarrow v_{m}(K)
$$

for $m \in \mathscr{L}_{-}$and that

$$
\limsup _{n \rightarrow \infty} \lambda_{m}\left(K_{n}\right) \geq 0
$$

if $\mathscr{L}_{-}=\{1,2, \ldots, q\}$ and $m \geq q+1$. Assume first that $\mathscr{L}_{-}=\mathbb{N}$. We may then employ (20) for every natural number $m$. As $n+1-\ell \geq m$ and thus $\lambda_{n+1-\ell}\left(K_{n}\right) \geq$ $\lambda_{m}\left(K_{n}\right)$ for all sufficiently large $n$, we infer from (20) that

$$
\liminf _{n \rightarrow \infty} \lambda_{n+1-\ell}\left(K_{n}\right) \geq v_{m}(K) .
$$

Because $v_{m}(K) \rightarrow 0$ as $m \rightarrow \infty$, we obtain that

$$
\liminf _{n \rightarrow \infty} \lambda_{n+1-\ell}\left(K_{n}\right) \geq 0,
$$

which together with (19) yields that $\lambda_{n+1-\ell}\left(K_{n}\right) \rightarrow 0$. Finally, under the assumption that $\mathscr{L}_{-}=\{1,2, \ldots, q\}$ we can use (21) for $m=q+1$, and since $n+1-\ell \geq q+1$ and hence $\lambda_{n+1-\ell}\left(K_{n}\right) \geq \lambda_{q+1}\left(K_{n}\right)$ for all sufficiently large $n$, we arrive again at (22), which in conjunction with (19) shows that $\lambda_{n+1-\ell}\left(K_{n}\right) \rightarrow 0$. This completes the proof of (13) and (14). The proof of the remaining two relations (15) and (16) is completely analogous. 
Proof of Theorem 1.1. Lemma 3.1 tells us that the nonzero eigenvalues of $T_{n, \alpha}$ are related to those of $G_{n, \alpha}$ via

$$
\lambda_{n+1-\ell}\left(T_{n, \alpha}\right)=n \lambda_{n+1-\ell}\left(G_{n, \alpha}\right),
$$

and since $G_{n, \alpha}=n^{\alpha} K_{n, \alpha}$, it follows that

$$
\frac{\lambda_{n+1-\ell}\left(T_{n, \alpha}\right)}{n^{\alpha+1}}=\lambda_{n+1-\ell}\left(K_{n, \alpha}\right) .
$$

Lemma 3.3 now implies that $\lambda_{n+1-\ell}\left(K_{n, \alpha}\right) \neq 0$ for $\ell \in \mathscr{L}_{+}$whenever $n$ is sufficiently large. Thus, equality (23) is true for these $\ell$, and Lemma 3.3 then yields that

$$
\lambda_{n+1-\ell}\left(T_{n, \alpha}\right)=\mu_{\ell}\left(K_{\alpha}\right) n^{\alpha+1}+o\left(n^{\alpha+1}\right) .
$$

For the remaining $\ell$, we have either $\lambda_{n+1-\ell}\left(T_{n, \alpha}\right)=0$ or $\lambda_{n+1-\ell}\left(T_{n, \alpha}\right) \neq 0$, and in the last case (23) holds. Thus, again by Lemma 3.3,

$$
\lambda_{n+1-\ell}\left(T_{n, \alpha}\right) / n^{\alpha+1} \longrightarrow 0 .
$$

To pass from $T_{n, \alpha}$ to $T_{n}$, note that $T_{n}=T_{n, \alpha}+B_{n}$ with $B_{n}=T_{n}\left[b_{0}, b_{1}, \ldots, b_{n-1}\right]$ and $b_{k}=o\left(k^{\alpha}\right)$. By Weyl's theorem on the eigenvalues of Hermitian matrices,

$$
\left|\lambda_{j}\left(T_{n}\right)-\lambda_{j}\left(T_{n, \alpha}\right)\right| \leq\left\|T_{n}-T_{n, \alpha}\right\|=\left\|B_{n}\right\|
$$

for each $j$, and the norm of the Toeplitz matrix $B_{n}$ is at most

$$
\left|b_{0}\right|+2\left|b_{1}\right|+\ldots+2\left|b_{n-1}\right|=o\left(n^{\alpha+1}\right) .
$$

This proves (4) and (5). The proof of (6) and (7) is analogous.

Finally, suppose $a_{k}$ is of the form (1). The kernel (12) is $O(1 / n)$ uniformly in $(x, y) \in[0,1]^{2}$ and hence $\left\|K_{n}-K\right\|=O(1 / n)$. This allows us to refine (17) to

$$
\operatorname{dist}\left(M I+K_{n}, \mathcal{F}_{\ell-1}\right)=\operatorname{dist}\left(M I+K, \mathcal{F}_{\ell-1}\right)+O(1 / n) .
$$

Hence $\lambda_{n+1-\ell}\left(K_{n}\right)=\mu_{\ell}(K)+O(1 / n)$ and thus

$$
\lambda_{n+1-\ell}\left(T_{n, \alpha}\right)=\mu_{\ell}(K) n^{\alpha+1}+O\left(n^{\alpha}\right) .
$$

Since $b_{k}=O\left(k^{\alpha-1}\right)$, the sum (24) and therefore also $\left\|T_{n}-T_{n, \alpha}\right\|$ is $O\left(n^{\alpha}\right)$.

\section{Toeplitz versus circulant matrices}

If we border a Hermitian $n \times n$ matrix $A_{n}$ by an $n+1$ st row and an $n+1$ st column to an $(n+1) \times(n+1)$ Hermitian matrix $B_{n+1}$, then, by Cauchy's interlace theorem,

$$
\lambda_{k}\left(B_{n+1}\right) \leq \lambda_{k}\left(A_{n}\right) \leq \lambda_{k+1}\left(B_{n+1}\right) \text { for } k=1,2, \ldots, n .
$$


Extending $A_{n}$ in this way to a Hermitian $(2 n-1) \times(2 n-1)$ matrix $B_{2 n-1}$, we obtain

$$
\lambda_{k}\left(B_{2 n-1}\right) \leq \lambda_{k}\left(A_{n}\right) \leq \lambda_{k+n-1}\left(B_{2 n-1}\right) \quad \text { for } k=1,2, \ldots, n .
$$

A symmetric Toeplitz matrix $T_{n}$ may in this manner be extended to a symmetric $(2 n-1) \times(2 n-1)$ circulant matrix $C_{2 n-1}$. For example, starting with

$$
T_{4}=\left(\begin{array}{llll}
1 & a & b & c \\
a & 1 & a & b \\
b & a & 1 & a \\
c & b & a & 1
\end{array}\right)
$$

we may by successive symmetric bordering achieve the circulant matrix

$$
C_{7}=\left(\begin{array}{llll|lll}
1 & a & b & c & c & b & a \\
a & 1 & a & b & c & c & b \\
b & a & 1 & a & b & c & c \\
c & b & a & 1 & a & b & c \\
\hline c & c & b & a & 1 & a & b \\
b & c & c & b & a & 1 & a \\
a & b & c & c & b & a & 1
\end{array}\right) .
$$

The eigenvalues of the $N \times N$ circulant matrix with the first column

$$
\left(\begin{array}{llll}
b_{0} & b_{1} & \ldots & b_{N-1}
\end{array}\right)^{\top}
$$

are the values of $p(z) \stackrel{\text { def }}{=} b_{0}+b_{1} z+\cdots+b_{N-1} z^{N-1}$ at the $N$ roots of $z^{N}=1$. Thus, computing the eigenvalues of $C_{2 n-1}$ and using (25), we get estimates for the eigenvalues of $T_{n}$. To compute and estimate $p(z)$ for the canonical matrices $T_{n, 1}$ we need the following lemma.

Lemma 4.1. Let $n \geq 3$. If $z^{2 n-1}=1, z \neq 1$, and $z^{n}=e^{i \varphi}$, then

$$
\operatorname{Re}\left(z^{2}+2 z^{3}+\cdots+(n-2) z^{n-1}\right)=\frac{1}{2}-\frac{1}{4(1+\cos \varphi)} .
$$


Proof. For arbitrary $z \neq 1$,

$$
\begin{aligned}
z^{2} & +2 z^{3}+\cdots+(n-2) z^{n-1} \\
& =z^{2}\left(1+\cdots+z^{n-3}\right)+z^{3}\left(1+\cdots+z^{n-4}\right)+\cdots+z^{n-1} \cdot 1 \\
& =z^{2} \frac{1-z^{n-2}}{1-z}+z^{3} \frac{1-z^{n-3}}{1-z}+\cdots+z^{n-1} \frac{1-z}{1-z} \\
& =\frac{z^{2}}{1-z}\left(1+\cdots+z^{n-3}\right)-\frac{(n-2) z^{n}}{1-z} \\
& =\frac{z^{2}\left(1-z^{n-2}\right)}{(1-z)^{2}}-\frac{(n-2) z^{n}}{1-z} \\
& =\frac{z^{2}-z^{n}}{(1-z)^{2}}-(n-2) \frac{z^{n}}{1-z} .
\end{aligned}
$$

Now suppose $z^{2 n-1}=1$, put $w=z^{n}$, and let $w=e^{i \varphi}$. Clearly,

$$
w^{2}=z^{2 n}=z
$$

Consequently,

$$
\frac{z^{n}}{1-z}=\frac{w}{1-w^{2}}=\frac{e^{i \varphi}}{1-e^{2 i \varphi}}=\frac{e^{i \varphi}\left(1-e^{-2 i \varphi}\right)}{\left|1-e^{2 i \varphi}\right|^{2}}=\frac{2 i \sin \varphi}{\left|1-e^{2 i \varphi}\right|^{2}}
$$

which shows that

$$
\operatorname{Re}\left(\frac{z^{n}}{1-z}\right)=0
$$

Furthermore,

$$
\begin{aligned}
\frac{z^{2}-z^{n}}{(1-z)^{2}} & =\frac{w^{4}-w}{\left(1-w^{2}\right)^{2}}=\frac{\left(e^{4 i \varphi}-e^{i \varphi}\right)\left(1-e^{-2 i \varphi}\right)^{2}}{\left|1-e^{2 i \varphi}\right|^{4}} \\
& =\frac{e^{2 i \varphi}\left(e^{2 i \varphi}-e^{-i \varphi}\right)\left(e^{-i \varphi}\left(e^{i \varphi}-e^{-i \varphi}\right)\right)^{2}}{\left((1-\cos 2 \varphi)^{2}+\sin ^{2} 2 \varphi\right)^{2}}
\end{aligned}
$$


and taking into account that $e^{i \varphi}-e^{-i \varphi}=2 i \sin \varphi$, we obtain

$$
\begin{aligned}
\operatorname{Re} \frac{z^{2}-z^{n}}{(1-z)^{2}} & =\frac{4 \sin ^{2} \varphi(\cos \varphi-\cos 2 \varphi)}{\left(1-2\left(1-2 \sin ^{2} \varphi\right)+1\right)^{2}} \\
& =\frac{1}{4} \frac{\cos \varphi-\cos 2 \varphi}{\sin ^{2} \varphi} \\
& =\frac{1}{4} \frac{\cos \varphi-1+2 \sin ^{2} \varphi}{\sin ^{2} \varphi} \\
& =\frac{1}{4}\left(2-\frac{1-\cos \varphi}{1-\cos ^{2} \varphi}\right) \\
& =\frac{1}{4}\left(2-\frac{1}{1+\cos \varphi}\right) .
\end{aligned}
$$

This implies the asserted formula.

Proof of Theorem 1.2(b). We extend $T_{n, 1}$ to the circulant matrix $C_{2 n-1}$ whose first column is

$$
\left(\begin{array}{lllllll}
0 & 1 & \ldots & n-1 & n-1 & \ldots & 1
\end{array}\right)^{\top} .
$$

Thus, the polynomial $p(z)$ may be written as

$$
\begin{aligned}
p(z)=z+z^{2}+\cdots+z^{2 n-2}+ & \left(z^{2}+2 z^{3}+\cdots+(n-2) z^{n-1}\right) \\
& +\left(z^{2 n-3}+2 z^{2 n-4}+\cdots+(n-2) z^{n}\right) .
\end{aligned}
$$

Obviously, $p(1)=n(n-1)$. So let $z^{2 n-1}=1$ and $z \neq 1$. Then

$$
\begin{gathered}
z+z^{2}+\cdots+z^{2 n-2}=-1, \\
z^{2 n-3}=\bar{z}^{2}, \quad z^{2 n-4}=\bar{z}^{3}, \quad \ldots, \quad z^{n}=\bar{z}^{n-1},
\end{gathered}
$$

and hence

$$
p(z)=-1+2 \operatorname{Re}\left(z^{2}+3 z^{3}+\cdots+(n-2) z^{n-1}\right) .
$$

From Lemma 4.1 we infer that

$$
p(z)=-\frac{1}{2} \frac{1}{1+\cos \varphi},
$$

where $z^{n} \stackrel{\text { def }}{=} e^{i \varphi}$, and since $1+\cos \varphi \leq 2$, it follows that $p(z) \leq-1 / 4$. Consequently,

$$
\lambda_{2 n-1}\left(C_{2 n-1}\right)=p(1)=n(n-1), \quad \lambda_{2 n-2}\left(C_{2 n-1}\right) \leq-1 / 4 .
$$

Due to (25), this implies that $\lambda_{n-1}\left(T_{n, 1}\right) \leq-1 / 4$. 
Remark 4.2. Let $F: \mathbb{R} \rightarrow \mathbb{R}$ be a continuous function with compact support. If $\alpha$ is an even integer, then Theorem 1.4 implies that the $p+q=\alpha+1$ extreme eigenvalues of $T_{n, \alpha}$ are eventually outside the support of $F$ and hence

$$
\lim _{n \rightarrow \infty} \frac{1}{n} \sum_{k=1}^{n} F\left(\lambda_{k}\left(T_{n, \alpha}\right)\right)=F(0) .
$$

By Theorem 1.2(b), this cannot be true for $\alpha=1$. We claim that if $\alpha=1$, then (28) does also not hold with $F(0)$ replaced by $F(s)$ for any $s \neq-1 / 2$. Assume the contrary. Clearly, we may suppose that $s \leq-1 / 4$. Take an $F$ so that $F(x) \leq F(y)$ for $-\infty \leq x \leq y \leq-1 / 4$. Put

$$
C_{F}=\liminf _{n \rightarrow \infty} \frac{1}{n} \sum_{k=1}^{n} F\left(\lambda_{k}\left(T_{n, 1}\right)\right),
$$

and

$$
D_{F}=\limsup _{n \rightarrow \infty} \frac{1}{n} \sum_{k=1}^{n} F\left(\lambda_{k}\left(T_{n, 1}\right)\right) .
$$

The proof of Theorem 1.2(b) shows that the eigenvalues of $C_{2 n-1}$ are $p(1)=n(n-1)$ and the values of $-1 /(2+2 \cos \varphi)$ at $\varphi=2 \pi j n /(2 n-1)$ for $1 \leq j \leq 2 n-2$. By the interlace property,

$$
\lambda_{k}\left(C_{2 n-1}\right) \leq \lambda_{k}\left(T_{n, 1}\right) \leq \lambda_{k+n-1}\left(C_{2 n-1}\right),
$$

and we also know that $\lambda_{2 n-2}\left(C_{2 n-1}\right) \leq-1 / 4$. Therefore

$$
F\left(\lambda_{k}\left(C_{2 n-1}\right)\right) \leq F\left(\lambda_{k}\left(T_{n, 1}\right)\right) \leq F\left(\lambda_{k+n-1}\left(C_{2 n-1}\right)\right)
$$

for $k \leq n-1$. It follows that

$$
\begin{aligned}
& C_{F} \geq \liminf _{n \rightarrow \infty} \frac{1}{n} \sum_{k=1}^{n} F\left(\lambda_{k}\left(C_{2 n-1}\right)\right), \\
& D_{F} \leq \limsup _{n \rightarrow \infty}\left(\frac{1}{n} \sum_{k=1}^{n-1} F\left(\lambda_{k+n-1}\left(C_{2 n-1}\right)\right)+\frac{1}{n} F\left(\lambda_{n}\left(T_{n, 1}\right)\right),\right.
\end{aligned}
$$

and interpreting the sums as integral sums one gets after some computations

$$
\begin{aligned}
\int_{0}^{1} F\left(-\frac{1}{2} \frac{1}{1-\cos (\pi x / 2)}\right) d x & \leq C_{F} \\
& \leq D_{F} \\
& \leq \int_{0}^{1} F\left(-\frac{1}{2} \frac{1}{1+\cos (\pi x / 2)}\right) d x .
\end{aligned}
$$


For $s>-1 / 2$, choose $\delta>0$ so that $-1 / 2+\delta<s$. Taking a function $F$ such that $F(s)=1$ and $F(y)=0$ for $y \leq-1 / 2+\delta$, we obtain

$$
\begin{aligned}
D_{F} & \leq \int_{1 /(2+2 \cos (\pi x / 2)) \leq 1 / 2-\delta} F\left(-\frac{1}{2} \frac{1}{1+\cos (\pi x / 2)}\right) d x \\
& \leq \int_{1 /(2+2 \cos (\pi x / 2)) \leq 1 / 2-\delta} d x \\
& =\left|\left\{x \in(0,1): \frac{1}{2+2 \cos (\pi x / 2)} \leq \frac{1}{2}-\delta\right\}\right|<1,
\end{aligned}
$$

which shows that the equality $D_{F}=F(s)$ is not valid. If $s<-1 / 2$, then there is a $\delta>0$ such that $s<-1 / 2-\delta$ and a function $F$ satisfying $F(s)=0$ and $F(-1 / 2-\delta)=1$. It follows that

$$
C_{F} \geq \int_{1 /(2-2 \cos (\pi x / 2)) \geq 1 / 2+\delta} d x>0,
$$

contradicting the inequality $0=F(s) \geq C_{F}$. We conjecture that $s=-1 / 2$ is also impossible.

Proof of Theorem 1.3(a). We extend $T_{n}$ to the circulant matrix $C_{2 n-1}$ with the first column

$$
\left(\begin{array}{lllllll}
R & R-h & \ldots & R-(n-1) h & R-(n-1) h & \ldots & R-h
\end{array}\right)^{\top} .
$$

The polynomial $p(z)$ now becomes

$$
\begin{aligned}
R+(R-h)\left(z+z^{2}+\cdots+z^{2 n-2}\right) & -h\left(z^{2}+2 z^{3}+\cdots+(n-2) z^{n-1}\right) \\
& -h\left(z^{2 n-3}+2 z^{2 n-4}+\cdots+(n-2) z^{n}\right) .
\end{aligned}
$$

We have $p(1)=R(2 n-1)-h n(n-1)=s_{n}$, and if $z^{2 n-1}=1, z \neq 1, z^{n} \stackrel{\text { def }}{=} e^{i \varphi}$, then (26), (27), and Lemma 4.1 show that

$$
\begin{aligned}
p(z) & =R+(R-h)(-1)-2 h \operatorname{Re}\left(z^{2}+3 z^{3}+\cdots+(n-2) z^{n-1}\right) \\
& =h-2 h\left(\frac{1}{2}-\frac{1}{4(1+\cos \varphi)}\right) \\
& =\frac{h}{2} \frac{1}{1+\cos \varphi} \geq \frac{h}{4} .
\end{aligned}
$$

Thus, if $p(1)=s_{n} \geq h / 4$, then all eigenvalues of $C_{2 n-1}$ are greater than or equal to $h / 4$, and we may deduce from (25) that $\lambda_{1}\left(T_{n}\right) \geq \lambda_{1}\left(C_{2 n-1}\right) \geq h / 4$.

Remark 4.3. To come back to Figure 2, we note that in the preceding proof we had not to consider $p(z)$ for all $z$ on the unit circle but only for the $2 n-1$ st unit roots. 


\section{Eigenvalues of integral operators}

Proof of Theorem 1.2(a). Let $\lambda \neq 0$ be a real number. This number is an eigenvalue of the integral operator $K_{1}$ if and only if

$$
\lambda f(x)=\int_{0}^{x}(x-y) f(y) d y+\int_{x}^{1}(y-x) f(y) d y
$$

for some function $f \in L^{2}(0,1)$ which does not vanish identically. The right-hand side of (29) is differentiable almost everywhere, and hence so also is $f$. Differentiating (29) we obtain

$$
\lambda f^{\prime}(x)=\int_{0}^{x} f(y) d y-\int_{x}^{1} f(y) d y .
$$

The right-hand side and thus also $f^{\prime}$ are again differentiable almost everywhere, and taking derivatives in (30) we arrive at the equality $\lambda f^{\prime \prime}(x)=2 f(x)$. Consequently,

$$
f(x)=A \cos \omega x+B \sin \omega x
$$

where $\omega \in(0, \infty) \cup i(0, \infty)$ satisfies $\omega^{2}=-2 / \lambda$. Inserting (31) in (29) we obtain after some computation that

$$
\begin{aligned}
-\frac{2}{\omega^{2}} f(x)=x\left(-\frac{A \sin \omega}{\omega}+\frac{B}{\omega}+\frac{B \cos \omega}{\omega}\right) & +A\left(\frac{1}{\omega^{2}}+\frac{\sin \omega}{\omega}+\frac{\cos \omega}{\omega^{2}}\right) \\
& +B\left(-\frac{\cos \omega}{\omega}+\frac{\sin \omega}{\omega^{2}}\right)-\frac{2}{\omega^{2}} f(x),
\end{aligned}
$$

which holds for almost all $x$ if and only if

$$
\begin{gathered}
A(-\sin \omega)+B(1+\cos \omega)=0, \\
A(1+\omega \sin \omega+\cos \omega)+B(\sin \omega-\omega \cos \omega)=0 .
\end{gathered}
$$

For this system to have a nontrivial solution $A, B$ it is necessary and sufficient that

$$
\operatorname{det}\left(\begin{array}{cc}
-\sin \omega & 1+\cos \omega \\
1+\omega \sin \omega+\cos \omega & \sin \omega-\omega \cos \omega
\end{array}\right)=-2-2 \cos \omega-\omega \sin \omega=0 .
$$

The equation $2+2 \cos \omega+\omega \sin \omega=0$ has countably many solutions $\omega_{1}<\omega_{2}<\ldots$ on the positive real half-line $(0, \infty)$ and exactly one solution $i \omega_{0}$ on the positive imaginary half-line $i(0, \infty)$. It is easily seen that $\omega_{k}=k \pi$ if $k$ is odd and that the positive solution of the equation

$$
2+2 \cos i \omega+i \omega \sin i \omega=2+2 \cosh \omega-\omega \sinh \omega=0
$$


is $\omega_{0}=2.40035 \ldots$. Thus, the set of the eigenvalues $\lambda=-2 / \omega^{2}$ of $K_{1}$ is exactly as asserted in Theorem 1.2(b). Finally, the rank of the matrix

$$
\left(\begin{array}{cc}
-\sin \omega & 1+\cos \omega \\
1+\omega \sin \omega+\cos \omega & \sin \omega-\omega \cos \omega
\end{array}\right)
$$

is 1 for $\omega \in\left\{i \omega_{0}, \omega_{2}, \omega_{4}, \ldots\right\}$ because then $\sin \omega \neq 0$ and it is 1 for $\omega \in\left\{\omega_{1}, \omega_{3}, \ldots\right\}$ because in that case $\sin \omega-\omega \cos \omega \neq 0$. It follows that the solutions $(A, B)$ of (32), (33) always span a one-dimensional space, and hence all eigenvalues have the multiplicity 1 .

Remark 5.1. The method employed to prove Theorems 1.1 and 1.2(a) works for many other types of Hermitian matrices as well, for instance, for Toeplitz-plusHankel matrices. Let $T_{n}=\left(a_{i j}\right)_{i, j=0}^{n-1}$ where $a_{i j}=u P(|i-j|)+v Q(i+j)$ with nonzero real numbers $u, v$, positive real numbers $\alpha, \beta, P(k)=k^{\alpha}+o\left(k^{\alpha}\right)$, and $Q(k)=k^{\beta}+o\left(k^{\beta}\right)$. In addition to the operator $K_{\alpha}$ given by (2), we now encounter the integral operator $L_{\beta}$ defined by

$$
\left(L_{\beta} f\right)(x)=\int_{0}^{1}(x+y)^{\beta} f(y) d y, \quad x \in(0,1),
$$

which in this context appeared already in [27]. Put $K=u K_{\alpha}$ if $\alpha>\beta, K=v L_{\beta}$ if $\alpha<\beta$, and $K=u K_{\alpha}+v K_{\beta}$ if $\alpha=\beta$. Then the conclusions of Theorem 1.1 remain true with $n^{\alpha+1}$ replaced by $n^{\max (\alpha, \beta)+1}$ and with $\mu_{\ell}\left(K_{\alpha}\right)$ and $v_{\ell}\left(K_{\alpha}\right)$ replaced by the positive eigenvalues $\mu_{\ell}(K)$ and the negative eigenvalues $v_{\ell}(K)$ of $K$. For example, the operator $K_{1}+L_{1}$ has exactly one positive eigenvalue, $\mu_{1}\left(K_{1}+L_{1}\right)=2 / \omega_{0}^{2}$ where $\omega_{0}$ is the positive solution of the equation $\cosh \omega-\omega \sinh \omega=0$, and countably many negative eigenvalues, $v_{k}\left(K_{1}+L_{1}\right)=-2 / \omega_{k}^{2}$ where $\omega_{k}$ ranges over the positive solutions of the equation $\cos \omega+\omega \sin \omega=0$.

Proof of Theorem 1.3(b). We have $T_{n}=R E-h T_{n, 1}$ where $E$ is the matrix all entries of which are 1. A theorem by Weyl states that if $A, B$ are Hermitian matrices, then their equally ordered eigenvalues satisfy $\left|\lambda_{j}(A)-\lambda_{j}(B)\right| \leq\|A-B\|$. Since $\|R E\|=R n$, we deduce from Weyl's theorem that

$$
\begin{aligned}
\lambda_{j}\left(T_{n}\right) & =\lambda_{j}\left(-h T_{n, 1}\right)+O(n) \\
& =-h \lambda_{n+1-j}\left(T_{n, 1}\right)+O(n)
\end{aligned}
$$

for all $j$. But from Theorems 1.1 and 1.2(a) we know that

$$
\begin{aligned}
\lambda_{1}\left(T_{n, 1}\right) & =v_{1}\left(K_{1}\right) n^{2}+O(n) \\
& =-\frac{2}{\pi^{2}} n^{2}+O(n),
\end{aligned}
$$


and

$$
\begin{aligned}
\lambda_{n}\left(T_{n, 1}\right) & =\mu_{1}\left(K_{1}\right) n^{2}+O(n) \\
& =\frac{2}{\omega_{0}^{2}} n^{2}+O(n) .
\end{aligned}
$$

This completes the proof.

The following proof of Theorem 1.4 is different from (and in our opinion simpler than) Trench's proof in [19] but has recourse to that proof when dealing with the ranks of $M_{\alpha}$ and $T_{n, \alpha}$.

Proof of Theorem 1.4. If $\alpha$ is an even integer, then $|x-y|^{\alpha}=(x-y)^{\alpha}$. Consequently, a real number $\lambda \neq 0$ is an eigenvalue for $K_{\alpha}$ if and only if there is a nontrivial $f \in L^{2}(0,1)$ such that

$$
\begin{aligned}
\lambda f(x) & =\int_{0}^{1}(x-y)^{\alpha} f(y) d y \\
& =\sum_{j=0}^{\alpha}(-1)^{\alpha-j}\left(\begin{array}{c}
\alpha \\
j
\end{array}\right) x^{j} \int_{0}^{1} y^{\alpha-j} f(y) d y .
\end{aligned}
$$

The right-hand side is a polynomial in $x$, and hence $f$ is necessarily of the form $f(x)=f_{0}+f_{1}+\cdots+f_{\alpha} x^{\alpha}$. With this ansatz the equation becomes

$$
\begin{aligned}
\sum_{j=0}^{\alpha} x^{j} \lambda f_{j} & =\sum_{j=0}^{\alpha}(-1)^{\alpha-j}\left(\begin{array}{c}
\alpha \\
j
\end{array}\right) x^{j} \sum_{k=0}^{\alpha} f_{k} \int_{0}^{1} y^{\alpha-j} y^{k} d y \\
& =\sum_{j=0}^{\alpha} x^{j} \sum_{k=0}^{\alpha}(-1)^{\alpha-j}\left(\begin{array}{c}
\alpha \\
j
\end{array}\right) \frac{1}{\alpha+1-j+k} f_{k},
\end{aligned}
$$

and this equation has a nontrivial solution if and only if $\lambda$ is an eigenvalue of the matrix $M_{\alpha}$. Thus, the nonzero eigenvalues of $K_{\alpha}$ are just the eigenvalues of the $(\alpha+1) \times(\alpha+1)$ matrix $M_{\alpha}$.

The rank of $M_{\alpha}$ does not change when deleting the factor $(-1)^{\alpha-j}\left(\begin{array}{l}\alpha \\ j\end{array}\right)$ in the $j$-th column,

$$
\operatorname{rank} M_{\alpha}=\operatorname{rank}\left(\frac{1}{\alpha+1-j+k}\right)_{j, k=0}^{\alpha} .
$$

Applying the formula

$$
\operatorname{det}\left(\frac{1}{u_{j}+v_{k}}\right)=\frac{\prod_{j>k}\left(u_{j}-u_{k}\right)\left(v_{j}-v_{k}\right)}{\prod_{j, k}\left(u_{j}+v_{k}\right)}
$$


for Cauchy determinants with $u_{j}=\alpha+1-j$ and $v_{j}=j$ to the matrix on the right of (34), we see that the determinant of this matrix is nonzero. It follows that rank $M_{\alpha}=\alpha+1$, which implies that all eigenvalues of $M_{\alpha}$ are nonzero and that therefore $K_{\alpha}$ has exactly $\alpha+1$ nonzero eigenvalues. This completes the proof of part (a).

Formulas (8) and (9) are immediate from (4) and (6). A matrix of the form $\left(a_{j} b_{k}\right)$ has rank 1 . The $j, k$ entry of $T_{n, \alpha}$ is

$$
(j-k)^{\alpha}=\sum_{m=0}^{\alpha}(-1)^{\alpha-m}\left(\begin{array}{l}
\alpha \\
m
\end{array}\right) j^{m} k^{\alpha-m}
$$

and hence rank $T_{n, \alpha} \leq \alpha+1$ for all $n$. (We learned this argument from Eugene Tyrtyshnikov when he was visiting Chemnitz in May 2000.) From (8) and (9) we infer that $T_{n, \alpha}$ has $\alpha+1$ nonzero eigenvalues whenever $n$ is large enough. This shows that rank $T_{n, \alpha}=\alpha+1$ for $n \geq n_{0}(\alpha)$. Clearly, for these $n$ the zero eigenvalues are $\lambda_{j}\left(T_{n, \alpha}\right)$ with $q+1 \leq j \leq n-p$. Trench [19] was able to show that actually $n_{0}(\alpha)=\alpha+1$. This completes the proof of (b).

Remark 5.2. Let $\alpha$ be an odd natural number. After $\alpha+1$ times differentiating the equation $\lambda f(x)=\left(K_{\alpha} f\right)(x)$, which reads

$$
\lambda f(x)=\int_{0}^{x}(x-y)^{\alpha} f(y) d y+\int_{x}^{1}(y-x)^{\alpha} f(y) d y,
$$

we arrive at the differential equation $\lambda f^{(\alpha+1)}(x)=2 \alpha ! f(x)$. Writing $\lambda=2 \alpha ! / \omega^{\alpha+1}$ we see that $f$ must be of the form

$$
f(x)=\sum_{k=0}^{\alpha} A_{k} \exp \left(\omega e^{2 \pi i k /(\alpha+1)} x\right) .
$$

Inserting this in (35) we obtain a linear system for the unknown coefficients $A_{0}, \ldots, A_{\alpha}$. The matrix of the system is an $(\alpha+1) \times(\alpha+1)$ matrix $M_{\alpha}(\omega)$ which depends on $\omega$. The eigenvalues of $K_{\alpha}$ are given by $\lambda=2 \alpha ! / \omega^{\alpha+1}$ where $\omega$ ranges over the solutions of the equation det $M_{\alpha}(\omega)=0$ lying on $\alpha+1$ rays starting at the origin.

Proof of Theorem 1.5. The matrix $T_{n, \alpha}$ has nonnegative entries and is irreducible. The theorem of Perron and Frobenius therefore implies that $\lambda_{n}\left(T_{n, \alpha}\right)=\left\|T_{n, \alpha}\right\|$. Hence $\left|\lambda_{1}\left(T_{n, \alpha}\right)\right| \leq \lambda_{n}\left(T_{n, \alpha}\right)$, and dividing this inequality by $n^{\alpha+1}$ and then passing to the limit $n \rightarrow \infty$, we deduce from Theorem 1.1 that $\left|v_{1}\left(K_{\alpha}\right)\right| \leq \mu_{1}\left(K_{\alpha}\right)$.

The norm $\left\|K_{\alpha}\right\|$ does not exceed the Hilbert-Schmidt norm $\left\|K_{\alpha}\right\|_{2}$, and as

$$
\begin{aligned}
\left\|K_{\alpha}\right\|_{2}^{2} & =\int_{0}^{1} \int_{0}^{1}|x-y|^{2 \alpha} d x d y \\
& =\frac{1}{(\alpha+1)(2 \alpha+1)},
\end{aligned}
$$


we get the estimate $\mu_{1}\left(K_{\alpha}\right) \leq D_{\alpha}$. On the other hand, $\left\|K_{\alpha}\right\| \geq\left\|K_{\alpha} \mathbf{1}\right\| /\|\mathbf{1}\|$ where $\mathbf{1}$ is the function which is identically 1 . Obviously, $\|\mathbf{1}\|=1$. We have

$$
\begin{aligned}
\left\|K_{\alpha} \mathbf{1}\right\|^{2} & =\int_{0}^{1}\left(\int_{0}^{1}|x-y|^{\alpha} d y\right)^{2} d x \\
& =\frac{1}{(\alpha+1)^{2}} \int_{0}^{1}\left(x^{\alpha+1}+(1-x)^{\alpha+1}\right)^{2} d x,
\end{aligned}
$$

and this is just $C_{\alpha}^{2}$. Thus, $\mu_{1}\left(K_{\alpha}\right) \geq C_{\alpha}$.

Finally, to prove that $\mu_{1}\left(K_{\alpha}\right)$ is a monotonically decreasing function of $\alpha$, let $\alpha<\beta$. Again by Perron-Frobenius theory, the spectral radius $\lambda_{n}\left(T_{n, \beta}\right)$ of $T_{n, \beta}$ increases when any entries of the matrix increase. Since

$$
|j-k|^{\beta}=|j-k|^{\beta-\alpha}|j-k|^{\alpha} \leq(n-1)^{\beta-\alpha}|j-k|^{\alpha},
$$

we conclude that $\lambda_{n}\left(T_{n, \beta}\right) \leq(n-1)^{\beta-\alpha} \lambda_{n}\left(T_{n, \alpha}\right)$. Dividing this inequality by $n^{\beta+1}$ and passing to the limit $n \rightarrow \infty$, we obtain $\mu_{1}\left(K_{\beta}\right) \leq \mu_{1}\left(K_{\alpha}\right)$ by virtue of formula (4).

\section{References}

[1] A. Böttcher and P. Dörfler, On the best constants in inequalities of the Markov and Wirtinger types for polynomials on the half-line. Linear Algebra Appl. 430 (2009), 10571069. MR 2489378 Zbl 1162.41004

[2] A. Böttcher and P. Dörfler, Weighted Markov-type inequalities, norms of Volterra operators, and zeros of Bessel functions. Math. Nachr. 283 (2010), 40-57. MR 2598592 Zbl 1186.41004

[3] A. Böttcher and P. Dörfler, Inequalities of the Markov type for partial derivatives of polynomials in several variables. J. Integral Equations Appl. 23 (2011), 1-37. MR 2781136 Zbl 1221.41006

[4] A. Böttcher and S. M. Grudsky, Spectral Properties of Banded Toeplitz, Matrices. Society for Industrial and Applied Mathematics (SIAM), Philadelphia (PA), 2005. MR 2179973 Zbl 1089.47001

[5] A. Böttcher, S. M. Grudsky, and E. A. Maksimenko, Inside the eigenvalues of certain Hermitian Toeplitz band matrices. J. Comput. Appl. Math. 233 (2010), 2245-2264. MR 2577763 Zbl 1195.15009

[6] A. Böttcher and B. Silbermann, Introduction to Large Truncated Toeplitz Matrices. Springer-Verlag, New York, 1999. MR 1724795 Zbl 0916.15012

[7] A. Böttcher and J. Virtanen, Norms of Toeplitz matrices with Fisher-Hartwig symbols. SIAM J. Matrix Anal. Appl. 29 (2007), 660-671. MR 2318370 Zbl 1145.47023

[8] A. Böttcher and H. Widom, From Toeplitz eigenvalues through Green's kernels to higher-order Wirtinger-Sobolev inequalities. Oper. Theory Adv. Appl. 171 (2007), 73-87. MR 2308557 Zbl 1118.47016 
[9] U. Grenander and G. Szegő, Toeplitz Forms and Their Applications. University of California Press, Berkeley and Los Angeles, 1958. MR 0094840 Zbl 0080.09501

[10] M. Kac, W. L. Murdock, and G. Szegő, On the eigenvalues of certain Hermitian forms. J. Rational Mech. Anal. 2 (1953), 767-800. MR 0059482 Zbl 0051.30302

[11] A. Yu. Novosel'tsev and I. B. Simonenko, Dependence of the asymptotics of extreme eigenvalues of truncated Toeplitz matrices on the rate of attaining the extremum by the symbol. Algebra i Analiz 16 (2004), 146-152. English transl. St. Petersburg Math. J. 16 (2005), 713-718. MR 2090855 Zbl 1091.47024

[12] S. V. Parter, Extreme eigenvalues of Toeplitz forms and applications to elliptic difference equations. Trans. Amer. Math. Soc. 99 (1961), 153-192. MR 0120492 Zbl 0099.32403

[13] S. V. Parter, On the extreme eigenvalues of Toeplitz matrices. Trans. Amer. Math. Soc. 100 (1961), 263-276. MR 0138981 Zbl 0118.09802

[14] S. V. Parter, On the distribution of the singular values of Toeplitz matrices. Linear Algebra Appl. 80 (1986), 115-130. MR 0851935 Zbl 0601.15006

[15] S. Serra Capizzano, On the extreme spectral properties of Toeplitz matrices generated by $L^{1}$ functions with several minima/maxima. BIT 36 (1996), 135-142. MR 1431579 Zbl 0851.15008

[16] S. Serra Capizzano, On the extreme eigenvalues of Hermitian (block) Toeplitz matrices. Linear Algebra Appl. 270 (1998), 109-129. MR 1484077 Zbl 0892.15014

[17] L. F. Shampine, Some $L_{2}$ Markoff inequalities. J. Res. Nat. Bur. Standards, Sect. B 69 (1965), 155-158. MR 0193295 Zbl 0143.08702

[18] W. F. Trench, Asymptotic distribution of the spectra of a class of generalized KacMurdock-Szegó matrices. Linear Algebra Appl. 294 (1999), 181-192. MR 1693915 Zbl 0942.15006

[19] W. F. Trench, Spectral distribution of generalized Kac-Murdock-Szegő matrices. Linear Algebra Appl. 347 (2002), 251-273. MR 1899893 Zbl 1071.15011

[20] E. E. Tyrtyshnikov, New theorems on the distribution of eigen and singular values of multiplevel Toeplitz matrices. Dokl. Akad. Nauk 333 (1993), 00-303. English transl. Russian Acad. Sci. Dokl. Math. 48 (1994), no. 3, 524-528. MR 1258226Zbl 0827.15029

[21] E. E. Tyrtyshnikov, A unifying approach to some old and new theorems on distribution and clustering. Linear Algebra Appl. 232 (1996), 1-43. MR 1366576 Zbl 0841.15006

[22] E. E. Tyrtyshnikov and N. L. Zamarashkin, Toeplitz eigenvalues for Radon measures. Linear Algebra Appl. 343/344 (2002), 345-354. MR 1878949 Zbl 0995.15021

[23] H. Widom, On the eigenvalues of certain Hermitian operators. Trans. Amer. Math. Soc. 88 (1958), 491-522. MR 0098321 Zbl 0101.09202

[24] H. Widom, Extreme eigenvalues of translation kernels. Trans. Amer. Math. Soc. 100 (1961), 252-262. MR 0138980 Zbl 0197.10903

[25] H. Widom, Extreme eigenvalues of $N$-dimensional convolution operators. Trans. Amer. Math. Soc. 106 (1963), 391-414. MR 0145294 Zbl 0205.14603

[26] H. Widom, Rapidly increasing kernels. Proc. Amer. Math. Soc. 14 (1963), 501-506. MR 0151806 Zbl 0115.32502 
[27] H. Widom, Hankel matrices. Trans. Amer. Math. Soc. 121 (1966), 1-35. MR 0187099 Zbl 0148.12303

[28] N. L. Zamarashkin and E. E. Tyrtyshnikov, Distribution of eigen and singular values under relaxed requirements to the generating function. Math. Sb. 188 (1997), 83-92. English transl. in Sb. Math 188 (1997), 1191-1201. MR 1481397 Zbl 0898.15007

[29] P. Zizler, R. A. Zuidwijk, K. F. Taylor, and S. Arimoto, A finer aspect of eigenvalue distribution of selfadjoint band Toeplitz matrices. SIAM J. Matrix Anal. Appl. 24 (2002), 59-67. MR 1920552 Zbl 1020.15007

Received October 14, 2011

J. M. Bogoya, Pontificia Universidad Javeriana, Departamento de Matemáticas,

Bogotá D. C., Colombia

E-mail: jbogoya@javeriana.edu.co

A. Böttcher, Technische Universität Chemnitz, Fakultät für Mathematik, 09107 Chemnitz, Germany

E-mail: aboettch@mathematik.tu-chemnitz.de

S. M. Grudsky, CINVESTAV del I.P.N., Departamento de Matemáticas, Apartado Postal 14-740, 07000 Ciudad de México, Mexico

E-mail: grudsky@math.cinvestav.mx 